\title{
Clinical and genetic spectrum of interstitial lung disease in Chinese children associated with surfactant protein $\mathrm{C}$ mutations
}

Da Hong ${ }^{1 \dagger}$, Dan Dai ${ }^{2 \dagger}$, Jing Liu', Congcong Zhang ${ }^{1}$, Tingting Jin ${ }^{1}$, Yanyan Shi', Gaoli Jiang ${ }^{1}$, Mei Mei ${ }^{1}$, Libo Wang ${ }^{1}$ and Liling Qian ${ }^{1 *}$

\begin{abstract}
Background: Mutations in the surfactant protein C gene (SFTPC) result in interstitial lung disease (ILD). Our objective was to characterize clinical and genetic spectrum of ILD in Chinese children associated with SFTPC mutations.

Methods: Six Chinese children with ILD heterozygous for SFTPC mutations were included. Candidate genes responsible for surfactant dysfunction were sequenced by next-generation sequencing. Subclones of SFTPC with novel mutations were generated and transiently transfected into A549 cells. The functional characterization of mutant surfactant protein C (SP-C) was evaluated by Western blotting and immunofluorescence.

Results: The age of onset ranged from 7 days to 15 months. All cases required supplemental oxygen. Failure to thrive (5/6) was the most significant extra-pulmonary manifestation. Hydroxychloroquine was given as the longterm treatment of lung disease in four patients and two of them responded well. Three mutations were identified in six patients: four with 173T, one with D105G, one with Y113H. Mutations in three patients were inherited and three arised de novo. Western blotting revealed totally different band patterns between mutant SP-C (D105G and Y113H) and the wildtype. Immunofluorescence showed mutant SP-C (D105G) was scarcely trafficked to lamellar bodies but localized well to early endosomes, which was in marked contrast to the wildtype protein.
\end{abstract}

Conclusion: SFTPC mutations were an important cause of childhood ILD in Chinese population. I73T was a common SFTPC mutation in Chinese ILD children associated with surfactant protein C mutations.

Keywords: Interstitial lung diseases, Surfactant protein C, Mutation, Chinese

\section{Background}

Interstitial lung disease (ILD) in children represents a heterogeneous group of respiratory disorders that are characterized by impaired gas exchange and diffuse infiltrates [1]. In children, ILD is most frequently diagnosed in the first year of life with a predominance of genetic entities [2]. In the past decade, significant advances have been made in understanding the underlying causes for childhood ILD (chILD) such as genetic disorders of surfactant dysfunction which result from mutations in

\footnotetext{
* Correspondence: Ilqian@126.com

${ }^{\dagger}$ Da Hong and Dan Dai contributed equally to this work.

'Department of Respiratory Medicine, Children's Hospital of Fudan University, No.399 Wanyuan Road, Shanghai 201102, China

Full list of author information is available at the end of the article
}

genes critical for the function and metabolism of pulmonary surfactant [3]. Among these genes is the surfactant protein $\mathrm{C}$ gene (SFTPC) located on chromosome $8 \mathrm{p} 21$. Its encoding protein, surfactant protein C (SP-C), is a hydrophobic 35-amino-acid polypeptide secreted into the alveolar space by alveolar type II epithelial cells to help reduce surface tension [4]. Since Nogee et al. first reported a case caused by an SFTPC mutation in 2001 [5], more than 60 mutations in SFTPC have been identified in pediatric ILD patients to date.

Lung disease caused by different SFTPC mutations varies greatly, from respiratory distress syndrome (RDS) in neonates to ILD in adults $[6,7]$. However, up to now a large proportion of the reported cases are of Caucasian or African descent. Only a few cases with Asian origin

(C) The Author(s). 2019 Open Access This article is distributed under the terms of the Creative Commons Attribution 4.0 International License (http://creativecommons.org/licenses/by/4.0/), which permits unrestricted use, distribution, and 
were reported [8-10]. Whether patients with different geographic and ethnic origins differ in clinical and genetic spectrum remains unclear. With the increase of awareness of this disease and advances in diagnostic technique, although rare, we discover that SFTPC mutations account for a substantial proportion of unexplained ILD with early onset in our Chinese population. Recently, Chen J et al. [11] reported 18 Chinese cases with surfactant dysfunction. Among the 15 patients who had SFTPC mutations, 5 different mutations were identified. However, the information regarding the genotype as well as the choice of treatments and therapeutic response of Chinese patients is still limited. Here, we report the clinical features and genetic findings in 6 Chinese subjects heterozygous for SFTPC mutations to expand the genetic and clinical spectrum.

\section{Methods}

\section{Patients}

In this study the subjects were identified among symptomatic infants and children who were clinically diagnosed as chILD and suspected of having genetic surfactant dysfunction and then referred for candidate gene sequencing in the laboratory at Children's Hospital of Fudan University between 2013 and 2018. According to an American Thoracic Society guideline [12], a child is regarded as having chILD if at least three of the following four criteria are present: (1) respiratory symptoms (cough, rapid and/or difficult breathing, or exercise intolerance); (2) respiratory signs (tachypnea, adventitious sounds, retractions, digital clubbing, failure to thrive, or respiratory failure); (3) hypoxemia; and (4) diffuse abnormalities on a chest radiograph or CT scan. Meanwhile, common diseases that can cause ILD were excluded as primary diagnosis by echocardiography and the screening of pathogens, autoimmune antibodies and immune deficiency. Clinical data were collected during the study. This study was approved by the ethics committees of Children's Hospital of Fudan University. Written informed consent was obtained from all parents or guardians of the patients.

\section{Genetic analysis}

Genomic DNA was isolated from blood of the patients and their parents using the QIAamp DNA Blood Mini kit (Qiagen, Hilden, Germany). Molecular analysis of the disease-causing genes $S F T P B, S F T P C, A B C A 3, N K X 2-1$, $C S F 2 R A$ and CSF2RB were performed through a self-designed gene panel using Ion Torrent PGM (Life Technologies). Targeted genomic regions covered exons and their flanking sequences of these six genes responsible for surfactant dysfunction. Library preparation was conducted by multiplex amplification using the Ion AmpliSeq Library Kit 2.0 (Life Technologies). Sequencing was performed using $316 \mathrm{v} 2$ chips (Life Technologies) on the Ion Torrent PGM platform. We use Torrent Suite software (Life Technologies) to compare base calls. Then we use NextGENe software (SoftGenetics) to read alignments and to call variants with the human reference genome hg19 (NCBI). The variants were then compared with dbSNP. Novel variants were analyzed with in silico tools MutationTaster, SIFT and PolyPhen2.

The validation of the variants was performed by PCR followed by direct Sanger sequencing using 3500XL Genetic Analyzer (Applied Biosystems).

\section{Functional analysis of SFTPC D105G mutation}

The methods used to characterize SFTPC D105G mutation such as SFTPC cDNA expression constructs, A549 cell line transfection, Western blotting and immunofluorescence were described previously [13].

For construction of mutant Flag/SP-C $C^{\text {D105G }}$, mutagenesis was performed by inverse PCR using KOD Plus Mutagenesis Kit (Toyobo, Japan) with pCDH-EGFP-Flag/SP-C ${ }^{\mathrm{WT}}$ serving as a template. The $5^{\prime}$ (forward) primer used for mutagenesis: GCTACCAGCAGCTGCTGATC. The 3' (reverse) primer: CATACACCACGAGGCCAGTG. All constructs were confirmed by Sanger sequencing.

\section{Results \\ Clinical presentation}

From 2013 to 2018, 45 patients were referred for candidate gene sequencing. The age at onset ranged from 0 month to 9 years. Twenty-six were males and 19 females. Six patients $(6 / 45,13.3 \%)$ with heterozygous SFTPC mutations were identified (Table 1). One patient (patient 3) has been reported previously [13], but was included into this study because of additional follow-up information. All 6 patients were of Chinese Han origin and born at term with uneventful pregnancy and delivery. There were three males and three females. The age at onset of patients with SFTPC mutations ranged from 7 days to 15 months (median 2.5 months) which seems to be earlier than those without surfactant dysfunction (median 1.2 years). There were no difference in symptoms and signs such as cough, tachypnea and cyanosis between patients with and without surfactant dysfunction. However, Failure to thrive was more prevalent in patients with SFPTC mutations (5/6) than those without surfactant dysfunction (12/39).

\section{Genetic results}

Out of all 6 patients, 4 patients carried the hot spot mutation I73T of which two were inherited and two arised de novo (Table 2). Patient 2 was the only one who had a family history of a brother with ILD at 1 year of life. In patient 5, we discovered a once reported mutation D105G [14]. His father and sister who carried the same 


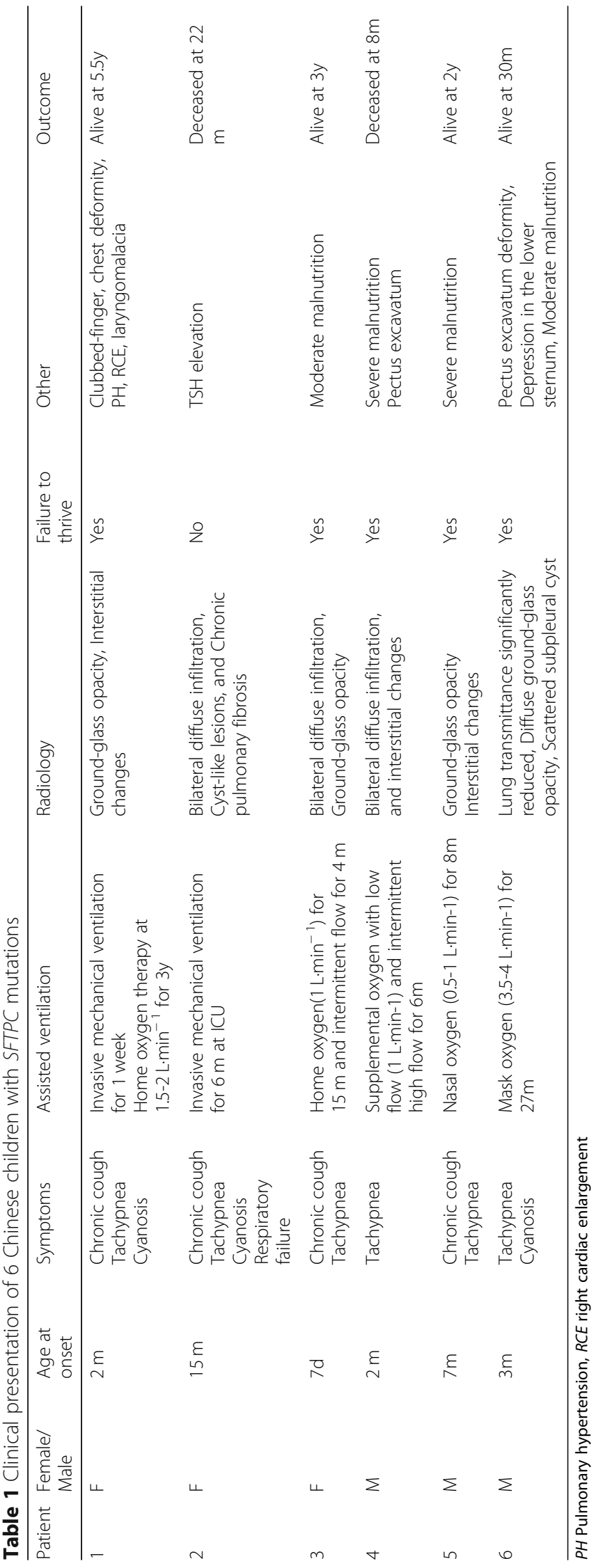


Table 2 Genetic information and family history of 6 patients with SFTPC mutations

\begin{tabular}{lllll}
\hline Patient & Mutation & De novo or inherited & SIFT/polyphen2 & Family history \\
\hline 1 & c.218T $>$ C, p.173T & Father I73T & Damaging/damaging & No \\
2 & c.218T $>$ C, p.I73T & $\begin{array}{l}\text { Mother I73T } \\
\text { Brother I73T }\end{array}$ & Damaging/damaging & Yes (brother diagnosed ILD at 1 years) \\
3 & c.337T > C, p.Y113H & De novo & Damaging/damaging & No \\
5 & c.218T $>$ C, p.173T & De novo & Damaging/damaging & No \\
6 & c.314A $>$ G, p.D105G & Father D105G & Damaging/damaging & No \\
\end{tabular}

mutation were asymptomatic. The patient 3 carrying a novel mutation $\mathrm{Y} 113 \mathrm{H}$ was described previously [13].

\section{Follow-up}

Empiric therapy such as corticosteroids, diuretics, antibiotics, aminophylline and cardiac stimulant were used when needed during exacerbations. Hydroxychloroquine was given as the long-term treatment of lung disease in four patients (Table 3). Two of them responded well with an ensuing catch-up of growth and withdrawal of oxygen supply. One (patient 6) responded partially with a decreased demand of oxygen, however weight gain was not remarkable due to repeated diarrhea caused by food allergy. As for the overall outcome, four patients survived with moderate or significant improvement while two died at 8 and 22 months respectively.

\section{Functional analysis of novel mutation}

The mutation D105G was only once reported by Willander et al. [14] However, in that report, the mutation carried by two ILD patients was inherited from their asymptomatic parents which was similar to our report. Moreover, no functional data of this mutation is currently available. Therefore, in order to determine the pathogenicity of the mutation, Western blotting and immunofluorescence were performed in A549 cells transfected with wild-type and D105G mutant SFTPC expression constructs.

To identify potential processing differences between proSP-C ${ }^{\mathrm{WT}}$, proSP-C $\mathrm{C}^{\mathrm{Y} 113 \mathrm{H}}$ and proSP-C $\mathrm{C}^{\mathrm{D} 105 \mathrm{G}}$, lysates of A549 cells transiently transfected with Flag/SP-C ${ }^{\mathrm{WT}}$, Flag/ SP-C $\mathrm{C}^{\mathrm{Y} 113 \mathrm{H}}$ and Flag/SP-C $\mathrm{C}^{\mathrm{D} 105 \mathrm{G}}$ expression constructs were analyzed by Western blotting. As is shown in Fig. 1, compared with proSP-C $\mathrm{C}^{\mathrm{WT}}$, multiple bands were missing for proSP-C $\mathrm{C}^{\mathrm{Y} 113 \mathrm{H}}$ and proSP-C $\mathrm{C}^{\mathrm{D} 105 \mathrm{G}}$. Meanwhile, an additional band at $20 \mathrm{kDa}$ was observed for proSP-C $\mathrm{C}^{\mathrm{D} 105 \mathrm{G}}$.

Immunofluorescence showed the intracellular localization of proproteins differed between A549 cells expressing proSP-C $\mathrm{C}^{\mathrm{WT}}$ and proSP-C $\mathrm{C}^{\mathrm{D} 105 \mathrm{G}}$ (Fig. 2). ProSP-C $\mathrm{C}^{\mathrm{WT}}$ was localized to CD63 (a marker for lamellar bodies and lysosomes)-positive and EEA1 (a marker for early endosome)negative vesicles, the expected target vesicle for the wild-type
proSP-C. On the contrary, proSP-C $\mathrm{C}^{\mathrm{D} 105 \mathrm{G}}$ hardly colocalized with CD63 but localized well with EEA1 indicating abnormal trafficking and accumulation in early endosomes.

Functioanal analysis of mutation $\mathrm{Y} 113 \mathrm{H}$ was described previously [13]. Briefly, in addition to different band patterns revealed by Western blotting as we mentioned above, ultrastructural analysis by transmission electron microscopy showed disorganized cytoplasmic organelles with hollow or eccentrically packed inclusions in cells expressing proSP-C $\mathrm{C}^{\mathrm{Y} 113 \mathrm{H}}$. Immunofluorescence demonstrated that proSP-C $\mathrm{C}^{\mathrm{Y} 113 \mathrm{H}}$ was scarcely trafficked to lamellar bodies but localized well to early endosomes, which was in marked contrast to the wild type protein.

\section{Discussion}

The identification of SFTPC mutations has led to significant advances in the diagnosis of interstitial lung disease in infancy and childhood. Due to the lack of diagnostic techniques, patients with SP-C dysfunction were frequently misdiagnosed in the past decades in China. In this study, we described 6 Chinese ILD patients with detailed clinical and genetic information which may help to provide a recognisable pattern for identifying such rare cases in clinical practice.

Most of the patients reported in our study had symptoms within the first year of life and then gradually developed dependence of oxygen with a finding of groundglass pattern on chest CT. This was consistent with other studies reported by pediatric centers in Western countries $[15,16]$. Our study showed an earlier age of onset and a more prevalent failure to thrive in group with SFTPC mutations. Referral for genetic analysis should be preferred in ILD patients with these features.

The severity of individuals with SFTPC mutations vary greatly, from severe RDS in neonates to mild interstitial lung disease in adults $[6,17,18]$. Clinical outcome at follow-up in our report varied from healthy (age 3 years) to deceased (age 8 and 22 months). Hydroxychloroquine has been reported to improve the clinical status of cases with SFTPC mutations. In some case series, 50 to $100 \%$ patients responded well to hydroxychloroquine treatment [16, 19, 20]. The exact mechanism of action of hydroxychloroquine 


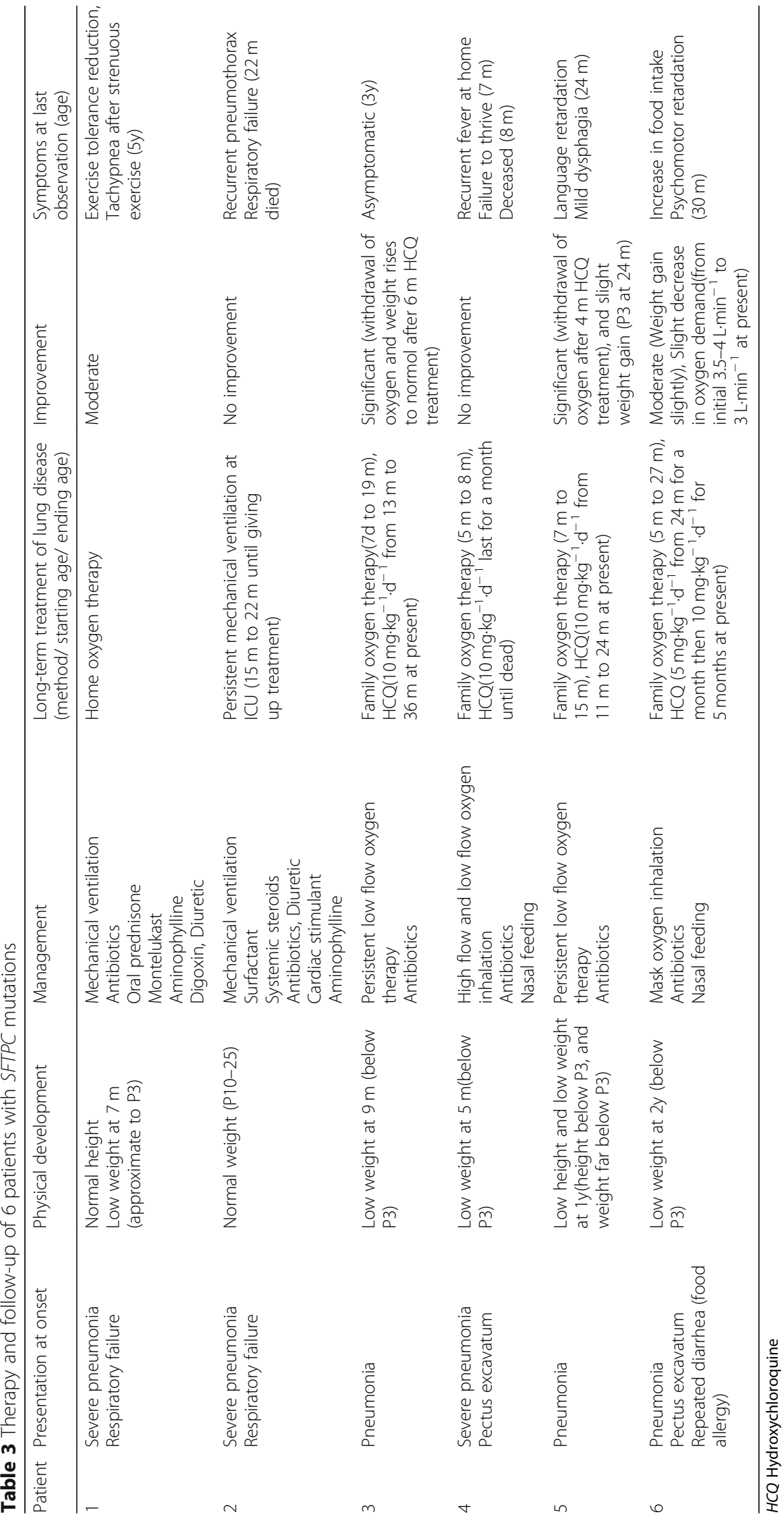




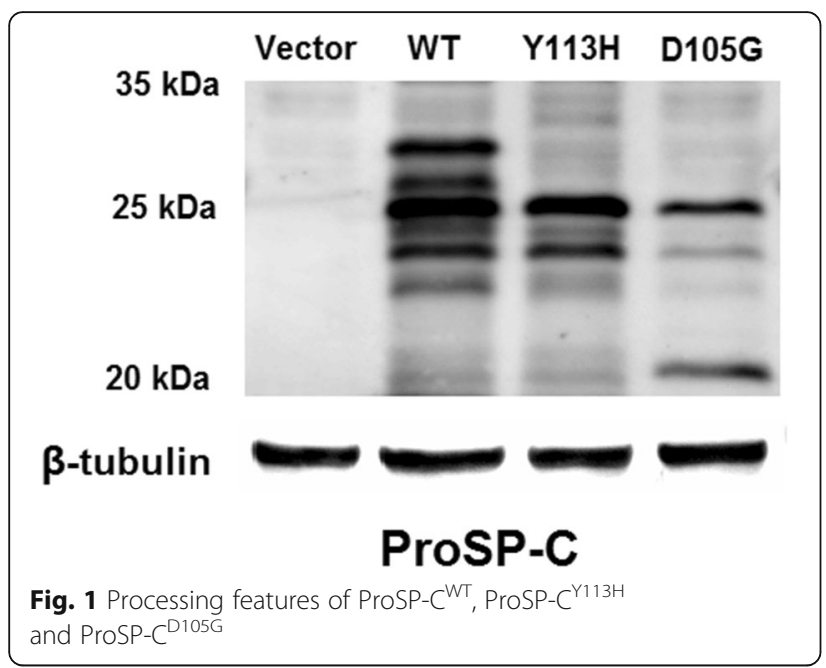

is unknown. In addition to having anti-inflammatory properties, hydroxychloroquine has been shown to cause inhibition of the intracellular processing of the precursor of SP-C [21], which may explain its therapeutic effects. $50 \%(2 / 4)$ of our patients responded well to hydroxychloroquine (initiated from 11 months and 13 months of life respectively) and one responded partially (initiated from 24 months of life). However, it should be noted that in the only case not

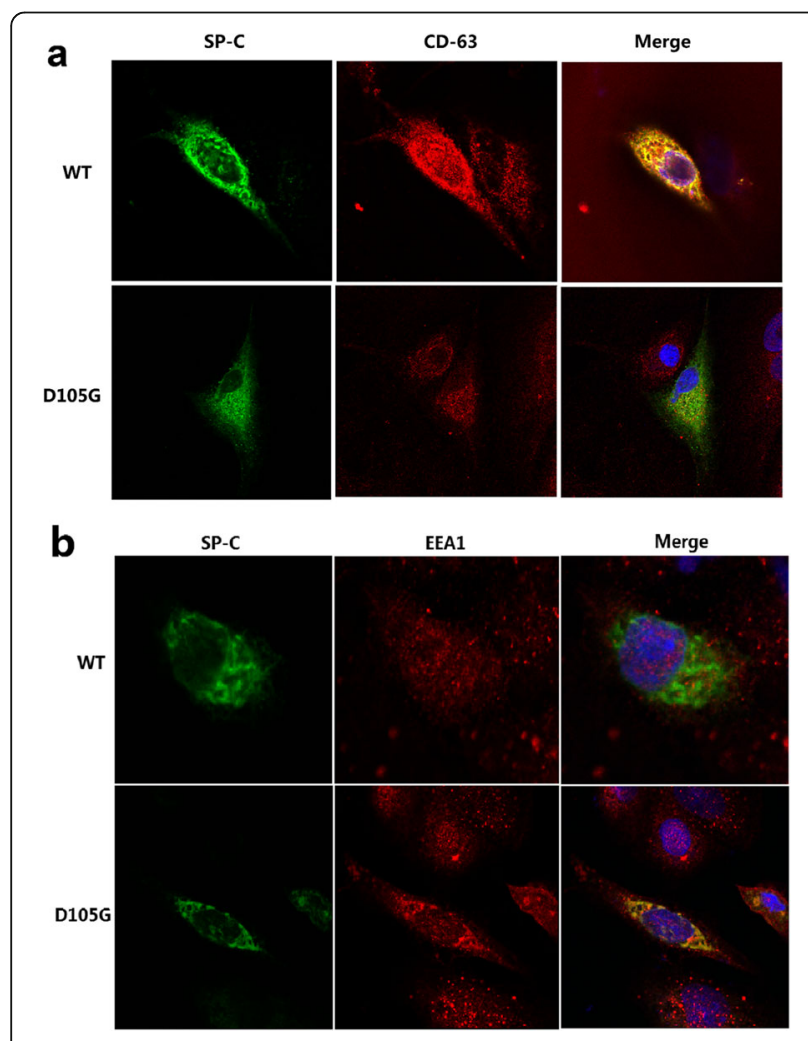

Fig. 2 Intracellular localization of ProSP-CWT and ProSP-C $C^{\text {D105G }}$ forms in transfected A549 cells responsive to hydroxychloroquine (initiated from 7 months of life), treatment had just begun for 1 month until he died of pulmonary exacerbation. Nowadays there are still few centers choosing hydroxychloroquine to treat ILD patients resulting from SFTPC mutations in China. In the future, more cases and longterm follow-up will be needed to determine the efficacy.

In terms of the genetic findings, we identified 3 different mutations in 6 patients, including two known and a novel mutations. I73T was the most common mutation accounting for $66.7 \%(4 / 6)$ of our patients which was consistent with other literature $(28-68 \%)[15,20,22]$. Mutations in half of the cases were inherited from parents and only one had family history suggesting imcomplete penetrance. The mechanism of imcomplete penetrance in this disease was still elusive. It was reported that heterozygosity for $A B C A 3$ (another gene responsible for surfactant dysfunction) mutations modifies the severity of lung disease in individuals with the same SFTPC mutation suggesting modifier genes may be involved [23]. In addition, Kaltenborn et al. [24] discovered infection with respiratory syncytial virus potentiated the mutational effects on loss of lung epithelial cell differentiation induced by $A B C A 3$ mutation. This study indicated that environmental factors such as viral infections may also have a key role in modulating the disease course thus contributing to the phenomenon of imcomplete penetrance.

The mutation D105G identified in patient 5 was once reported [14]. However, the father and sister of the patient who also carried the mutation showed no signs of any lung diseases leading us to questioning the pathogenicity of the mutation. Moreover, no functional data of this mutation is currently available. So together with the novel mutation $\mathrm{Y} 113 \mathrm{H}$, in vitro functional study was performed. According to previous research, many SFTPC mutations such as exon 4 deletion cause chronic accumulation of misfolded proSP$\mathrm{C}$ leading to endoplasmic reticulum (ER) stress and alveolar type II cell apoptosis [25, 26]. In our Western bloting analysis, multiple bands of proSP-C $\mathrm{C}^{\mathrm{Y} 113 \mathrm{H}}$ and proSP-C $\mathrm{C}^{\mathrm{D} 105 \mathrm{G}}$ were missing or significantly reduced when compared with the wild-type proprotein suggesting aberrant protein processing of both mutant proteins. However, an accumulated proprotein at $20 \mathrm{kDa}$ was observed for proSP-C $\mathrm{C}^{\mathrm{D} 105 \mathrm{G}}$ while not for proSP-C $\mathrm{C}^{\mathrm{Y} 113 \mathrm{H}}$ indicating distinct proprotein processing. Immunofluorescence assay of transfected A549 cells showed proSP-C ${ }^{\mathrm{Y} 113 \mathrm{H}}$ and proSP-C $\mathrm{C}^{\mathrm{D} 105 \mathrm{G}}$ both predominantly colocalized with EEA1 but not with lamellar body marker CD63. So we speculate, unlike proSP-C ${ }^{\mathrm{WT}}$ secreted via lamellar body fusion with the plasma membrane and then catabolized mainly by alveolar macrophages, misfolded proSP-C ${ }^{\mathrm{Y} 113 \mathrm{H}}$ and proSP-C $\mathrm{C}^{\mathrm{D} 105 \mathrm{G}}$ were endocytosed into endosome. ProSP- $\mathrm{C}^{\mathrm{D} 105 \mathrm{G}}$ may trigger the unfolded 
protein response and result in ER stress while proSP$\mathrm{C}^{\mathrm{Y} 113 \mathrm{H}}$ may be degraded by the ubiquitin-proteasome system as previously described [13]. The difference in molecular pathogenesis between D105G and Y113H may partially explain why imcomplete penetrance occured in family of patient 5 but not patient 3 .

A limitation of the study is that no lung samples of these patients were available. Therefore in vivo data regarding mutant SP-C expression and ultrastructure of alveolar type II cells were unclear. Besides, due to the rareness of this disease, our study is limited by small sample size and further multicenter study will be needed in the future. Also, a longer follow-up period is needed to determine the long-term outcome of the patients and the efficacy of the treatment.

\section{Conclusions}

Our study confirmed SFTPC mutations were an important cause of childhood ILD in the Chinese population. I73T was also a common mutation in Chinese ILD children. A part of patients with SFTPC mutations can benefit from hydroxychloroquine treatment. A further multi-center longitudinal study will be needed to investigate the phenotype-genotype association and treatment effect in Chinese population.

\section{Abbreviations}

HCQ: Hydroxychloroquine; ILD: Interstitial lung disease; PH: Pulmonary hypertension; RCE: Right cardiac enlargement; RDS: Respiratory distress syndrome; SFTPC: Surfactant protein C gene; SP-C: Surfactant protein C

\section{Acknowledgements}

We would like to thank all the parents who agreed to participate in the study.

\section{Authors' contributions}

$\mathrm{DH}$ and DD designed the study, drafted the manuscript, analyzed data and equally contributed to the work. JL, CZ, TJ, YS, GJ, MM and LW collected the data. LQ participated in the study design and coordinated the study. All authors read and approved the final manuscript.

\section{Funding}

This study is supported by grants from the National Natural Science Foundation of China (No.8167150), the Development Fund for Shanghai talents (201450)

\section{Availability of data and materials}

The datasets are available from the corresponding author on reasonable request.

\section{Ethics approval and consent to participate}

This study was approved by the ethics committees of Children's Hospital of Fudan University. Written informed consent was obtained from all parents or guardians of the patients.

\section{Consent for publication}

All the authors gave the consent for publication.

\section{Competing interests}

The authors declare that they have no competing interests.

\section{Author details}

'Department of Respiratory Medicine, Children's Hospital of Fudan University, No.399 Wanyuan Road, Shanghai 201102, China. ${ }^{2}$ Children's Hospital of Fudan University, No.399 Wanyuan Road, Shanghai 201102, China.

Received: 19 March 2019 Accepted: 21 August 2019

Published online: 28 August 2019

\section{References}

1. Clement A, Eber E. Interstitial lung diseases in infants and children. Eur Respir J. 2008;31:658-66.

2. Wert SE, Whitsett JA, Nogee LM. Genetic disorders of surfactant dysfunction. Pediatr Dev Pathol. 2009;12:253-74.

3. Nogee LM. Genetic basis of children's interstitial lung disease. Pediatr Allergy Immunol Pulmonol. 2010;23:15-24.

4. Whitsett JA, Weaver TE. Hydrophobic surfactant proteins in lung function and disease. N Engl J Med. 2002;347:2141-8.

5. Nogee LM, Dunbar AR, Wert SE, et al. A mutation in the surfactant protein C gene associated with familial interstitial lung disease. N Engl J Med. 2001; 344:573-9.

6. Thomas AQ, Lane K, Phillips JR, et al. Heterozygosity for a surfactant protein $C$ gene mutation associated with usual interstitial pneumonitis and cellular nonspecific interstitial pneumonitis in one kindred. Am J Respir Crit Care Med. 2002;165:1322-8.

7. Soraisham AS, Tierney AJ, Amin HJ. Neonatal respiratory failure associated with mutation in the surfactant protein C gene. J Perinatol. 2006;26:67-70.

8. Ono S, Tanaka T, Ishida M, et al. Surfactant protein C G100S mutation causes familial pulmonary fibrosis in Japanese kindred. Eur Respir J. 2011;38:861-9.

9. Akimoto T, Cho K, Hayasaka I, et al. Hereditary interstitial lung diseases manifesting in early childhood in Japan. Pediatr Res. 2014;76:453-8.

10. Huang L, Wang M, Chen Z, et al. I73T mutation in the pulmonary surfactant protein $C$ gene associated with pediatric interstitial lung disease: a case study and the review of related literature. Zhonghua Er Ke Za Zhi. 2014;52: 846-50.

11. Chen J, Nong G, Liu X, et al. Genetic basis of surfactant dysfunction in Chinese children: a retrospective study. Pediatr Pulmonol. 2019;54:11731181

12. Kurland G, Deterding RR, Hagood JS, et al. An official American Thoracic Society clinical practice guideline: classification, evaluation, and management of childhood interstitial lung disease in infancy. Am J Respir Crit Care Med. 2013;188:376-94.

13. Hong D, Qi Y, Liu J, et al. A novel surfactant protein C mutation resulting in aberrant protein processing and altered subcellular localization causes infantile interstitial lung disease. Pediatr Res. 2017;81:891-7.

14. Willander $\mathrm{H}$, Askarieh $\mathrm{G}$, Landreh $\mathrm{M}$, et al. High-resolution structure of a BRICHOS domain and its implications for anti-amyloid chaperone activity on lung surfactant protein C. Proc Natl Acad Sci U S A. 2012;109:2325-9.

15. Guillot L, Epaud R, Thouvenin G, et al. New surfactant protein C gene mutations associated with diffuse lung disease. J Med Genet. 2009;46:490-4.

16. Kroner C, Reu S, Teusch V, et al. Genotype alone does not predict the clinical course of SFTPC deficiency in paediatric patients. Eur Respir J. 2015; 46:197-206.

17. Poterjoy BS, Vibert $Y$, Sola-Visner M, et al. Neonatal respiratory failure due to a novel mutation in the surfactant protein C gene. J Perinatol. 2010;30:151-3.

18. Cameron HS, Somaschini M, Carrera P, et al. A common mutation in the surfactant protein C gene associated with lung disease. J Pediatr. 2005;146: $370-5$.

19. Avital A, Hevroni A, Godfrey S, et al. Natural history of five children with surfactant protein C mutations and interstitial lung disease. Pediatr Pulmonol. 2014;49:1097-105.

20. Thouvenin G, Abou TR, Flamein F, et al. Characteristics of disorders associated with genetic mutations of surfactant protein C. Arch Dis Child. 2010;95:449-54.

21. Beers MF. Inhibition of cellular processing of surfactant protein $C$ by drugs affecting intracellular pH gradients. J Biol Chem. 1996;271:14361-70.

22. Turcu $S$, Ashton $E$, Jenkins $L$, et al. Genetic testing in children with surfactant dysfunction. Arch Dis Child. 2013;98:490-5.

23. Bullard JE, Nogee LM. Heterozygosity for ABCA3 mutations modifies the severity of lung disease associated with a surfactant protein C gene (SFTPC) mutation. Pediatr Res. 2007;62:176-9. 
24. Kaltenborn E, Kern S, Frixel S, et al. Respiratory syncytial virus potentiates ABCA3 mutation-induced loss of lung epithelial cell differentiation. Hum Mol Genet. 2012;21:2793-806.

25. Mulugeta S, Nguyen V, Russo SJ, et al. A surfactant protein C precursor protein BRICHOS domain mutation causes endoplasmic reticulum stress, proteasome dysfunction, and caspase 3 activation. Am J Respir Cell Mol Biol. 2005;32:521-30

26. Mulugeta S, Maguire JA, Newitt JL, et al. Misfolded BRICHOS SP-C mutant proteins induce apoptosis via caspase-4- and cytochrome c-related mechanisms. Am J Physiol Lung Cell Mol Physiol. 2007;293:L720-9.

\section{Publisher's Note}

Springer Nature remains neutral with regard to jurisdictional claims in published maps and institutional affiliations.

Ready to submit your research? Choose BMC and benefit from:

- fast, convenient online submission

- thorough peer review by experienced researchers in your field

- rapid publication on acceptance

- support for research data, including large and complex data types

- gold Open Access which fosters wider collaboration and increased citations

- maximum visibility for your research: over $100 \mathrm{M}$ website views per year

At BMC, research is always in progress.

Learn more biomedcentral.com/submissions 